\title{
Early repatriation post-thrombectomy: a model of care which maximises the capacity of a stroke network to treat patients with large vessel ischaemic stroke
}

\author{
Emma Griffin, ${ }^{1,2}$ Sean Murphy, 2,3,4 Mark Sheehan, ${ }^{1}$ Sarah Power, ${ }^{1}$ Paul Brennan, ${ }^{1}$ \\ Alan O'Hare, ${ }^{1}$ Seamus Looby, ${ }^{1}$ Sebastian McWilliams, ${ }^{1}$ Barry Moynihan, ${ }^{5}$ \\ David Williams, ${ }^{2,5}$ Karl Boyle, ${ }^{5}$ Damien O'Neill, ${ }^{1}$ Ronan Collins, ${ }^{6}$ Eamon Dolan, ${ }^{7}$ \\ Tim Cassidy, ${ }^{8}$ Joe Harbison, ${ }^{9}$ Margaret $\mathrm{O}^{\prime}$ Connor, ${ }^{10}$ Jack Alderson, ${ }^{1,2}$ John Thornton ${ }^{1,2}$
}

For numbered affiliations see end of article.

\section{Correspondence to}

Dr Emma Griffin, Department of Radiology, Dublin, Ireland; emma.griffin.2@ucdconnect.ie

Received 5 December 2019 Revised 22 February 2020 Accepted 25 February 2020 Published Online First 15 April 2020

Check for updates

(C) Author(s) (or their employer(s)) 2020. No commercial re-use. See rights and permissions. Published by BMJ.

To cite: Griffin E, Murphy $\mathrm{S}$, Sheehan $\mathrm{M}$, et al.

J Neurolntervent Surg

2020;12:1166-1171.

\section{ABSTRACT}

Background and purpose Healthcare systems are challenged to provide access to thrombectomy in acute stroke patients. Either the "drip and ship" or "mothership" models result in increased numbers of patients in the endovascular stroke centre (ESC). We describe our approach for a "drip, ship, retrieve and leave" model repatriating patients immediately or within 24 hours of thrombectomy.

Methods We included consecutive patients who underwent thrombectomy from January 2016 to June 2018. Patients from local region primary stroke centres (PSC) are immediately repatriated and those from remote region PSCs are admitted for 24 hours before repatriation. Key parameters recorded included clinical, radiological and timeline data as well as 90-day modified Rankin Scale (mRS). Patients who stayed beyond the intended time period in the ESC were analysed.

Results From January 2016 to June 2018, 435 patients were transferred, with 352 patients in the local region group and 83 in the remote region group. The median NIHSS was 16 with a median ASPECTS of 9 . Good functional outcome was seen in $47 \%$ of patients with a mortality rate of $19 \%$. The local region group that were repatriated at the intended time period had a 90-day mRS $0-2$ of $47 \%$ compared with $20 \%$ of those admitted to the ESC $(P=0.006)$. Mortality rates were $20 \%$ and $27 \%$ respectively $(P=0.377)$. The remote region group, repatriated at 24 hours' post-emergency endovascular thrombectomy had 90 -day mRS $0-2$ of $65 \%$ compared with $41 \%$ in the group admitted $(P=0.042)$. Mortality rates were $4 \%$ and $22 \%$ respectively $(P=0.007)$. Conclusion This model enables the treatment of large numbers of patients with large vessel occlusion acute ischaemic stroke with thrombectomy within a national stroke service and system of care which would not otherwise be facilitated.

\section{INTRODUCTION}

Since the publication of several landmark randomised control trials in 2015, emergency endovascular thrombectomy (EVT) is now the standard of care in the management of patients presenting with acute ischaemic stroke (AIS) due to large vessel occlusion (LVO). ${ }^{1}$ Further clinical trials since then enrolling highly selected patients using advanced CT and MRI imaging have extended the time window for EVT up to 24 hours' postonset or last seen well. ${ }^{2} 3$ Early and rapid access to imaging and treatment has been shown to have a direct impact on positive functional outcomes for patients. ${ }^{45}$ Healthcare systems worldwide are grappling with how best to configure health service provision in acute stroke to enable timely availability of this highly efficacious therapy for patients who often have severe acute deficits. This treatment is confined to stroke centres with the capability for emergency endovascular care delivered by neuro-endovascular specialists. There has been much discussion and debate about different models of EVT provision such as 'drip and ship' vs 'mothership'. A number of studies using computational modelling have been published and results are awaited from the ongoing RACECAT randomised trial from Catalonia (NCT02795962). ${ }^{6}{ }^{7}$ Either way, significantly increased volumes of patients are now being admitted to endovascular stroke centres (ESC) for EVT, placing additional burdens on these centres and existing stroke networks services in terms of funding and workload. We describe a novel model whereby, following EVT, patients are systematically repatriated either immediately or within 24 hours of the thrombectomy procedure to the primary stroke centre (PSC), a system we term 'drip, ship, retrieve and leave'.

\section{METHODS}

Our institution provides a 24/7 emergency endovascular therapy for AIS LVO patients. It is the only centre within Ireland that provides a round-theclock EVT service. In addition to this, it is also the National Neurosurgical Centre. There are 24 hospitals in Ireland admitting patients with AIS for stroke unit care and which provide intravenous thrombolysis therapy. In addition to this, there are several private hospitals in the Greater Dublin Area from which we also receive patients directly for EVT. Due to a lack of available resources and capacity, as well as geographical distance it is not possible for 
all suspected Irish stroke cases to come directly to the ESC for initial assessment and hyperacute stroke treatment with thrombolysis. Therefore, patients beyond the immediate catchment area of the endovascular stroke centre are assessed, imaged and treated with intravenous thrombolysis in the nearest PSC. Those patients felt suitable for EVT are emergently transferred to the ESC for same. Following endovascular therapy, we have developed a pragmatic model whereby patients are repatriated to the PSC either immediately or within 24 hours following the thrombectomy procedure depending on their distance from the ESC and clinical stability immediately following treatment. Eligibility for EVT is based on agreed National Thrombectomy Service guidelines derived from current best evidence, which includes LVO either in the anterior circulation, ASPECTS $\geq 5$, NIHSS $>5$, presence of good collateral circulation particularly for those presenting $>6$ hours defined as $>50 \%$ of pial circulation and the possibility of revascularisation within 24 hours of stroke onset or last known well. ${ }^{12}$ Our institution has previously demonstrated good outcomes for patients presenting $>6$ and also $>12$ hours from symptom onset. ${ }^{89}$ Very few centres in Ireland use perfusion CT for thrombectomy case selection.

In the PSC, patients have clinical and radiological evaluation incorporating CT and CT angiography (CTA), either single or multiphase. They receive intravenous thrombolysis as appropriate. Following the diagnosis of LVO, contact is made directly with the interventional neuroradiology service in the ESC. The decision is made immediately whether or not to transfer for EVT. Patients are transported from the PSC to ESC, by paramedic crews, accompanied by medical and nursing personnel. Transfers are via ground transport in the vast majority of cases. On arrival at the ESC, immediate repeat clinical assessment is performed by the receiving neuroradiology team. If it has been more than 2 hours since the initial diagnostic imaging, a non-contrast CT scan is performed to assess for any possible progression of infarct or development of haemorrhage. Otherwise, the patient is transported immediately to the neuro-angiography suite where EVT is performed in a standard manner. In the majority of cases this is performed without general anaesthesia.

Our institution has moved to a standardised aspiration-first approach to thrombectomy from September 2017. Prior to this date, patients were treated primarily with stent retrievers. This is described elsewhere and has resulted in improved thrombolysis in cerebral infarction score (TICI) scores in fewer passes and shorter procedures. ${ }^{10}$ In brief, for the aspiration first approach, an 8 French short sheath is introduced to the common femoral artery. A 6 French Weinberg catheter (Balt Extrusion, Rue du Fonds des Aulnes, 95160 Montmorency, France) inside an 8 French Mach guide catheter (Boston Scientific, 100 Boston Scientific Way, Marlborough, MA 01752) is advanced to the aortic arch with a Terumo Glidewire 0.035 " (Terumo Medical Corporation Corporate Office 2101 Cottontail Lane Somerset, NJ 08873) and the relevant large vessel of the neck is catheterised. The 6-8 combination is then advanced into the internal carotid artery with the 8 French guide advanced over the 6 French catheter for final tip placement in the proximal to midinternal carotid artery. The wire and 6 French Weinberg are removed. Angiography confirms the site of vessel occlusion. A 6 Fr SOFIA Plus catheter (MicroVention, Inc. Worldwide Innovation Center 35 Enterprise Aliso Viejo, CA 92656) is advanced through a rotating haemostatic "Y" valve attached to a heparinised saline flush into the 8 French guide catheter. A $20 \mathrm{~mL}$ syringe partially filled with saline is attached to the hub of the catheter allowing back flow of blood in the absence of a wire within the catheter. Once the SOFIA Plus catheter extends beyond the tip of the 8 French guide catheter the $20 \mathrm{~mL}$ syringe is switched, following aspiration of any blood, for a $10 \mathrm{~mL}$ contrast filled syringe. Contrast is trickled forward as the SOFIA Plus catheter advances. Additional support/catheters may be required to advance the SOFIA Plus. Once the aspiration catheter reaches the thrombus, a $50 \mathrm{~mL}$ luer lock lockable syringe is then attached to the catheter and fixed suction is applied. If unsuccessful a stent retriever may be deployed. Prior to switching to this standardised approach, our regular approach consisted of using the same 8-6 access combination, an intermediate catheter in the distal ICA, crossing the occlusion with a microcatheter and using a stent retriever.

For the purposes of this study, patients are categorised into two groups: the local region group (LRG) who have arrived from hospitals within a 90-minute drive of the ESC and have a median distance of 43 kilometres $(6 \mathrm{~km}-137 \mathrm{~km})$ and the remote region group (RRG), greater than a 90 -minute drive, with a median distance of 217 kilometres $(181 \mathrm{~km}-244 \mathrm{~km})$.

Patients from the LRG are expected to be repatriated immediately following EVT, accompanied by the medical/nursing personnel. If the emergency crew has been able to wait, they are repatriated immediately by the same crew. If not, a new emergency ambulance crew is requested and the patient is transferred as soon as possible. For the RRG, they are admitted to the ESC stroke unit under the care of the stroke team, with the intention of being repatriated to the PSC within 24 hours. The criteria for patients who are not repatriated immediately are not specific and allow for clinical assessment and judgement. Decisions are made on a case-by-case basis by the neuro-interventional team in consultation with our inhouse stroke team. If there is clinical deterioration, perhaps due to haemorrhage, unsuccessful recanalisation or concern that further intervention (eg, craniectomy or early carotid stenting) or if immediate intensive care unit management is required, they would remain in the ESC. We do not use an NIHSS as criteria for keeping patients in the ESC. However, in general, if patients are stable or are improving, they are repatriated.

A system of governance is in place for this service through collaboration with stroke teams in PSCs and a standard pathway has been developed for this transfer process. Roles and responsibilities of personnel throughout the service as well as requirements for PSC and ESCs are established and agreed between centres. A prospective database is maintained of all aspects of this process under the remit of an ongoing service audit and is therefore excluded from the requirement for ethics approval. This ongoing audit of the service is registered with the Institutional Quality and Standards Department in the ESC.

Data collected includes patient demographics, all key time metrics, clinical and radiological findings, EVT procedural details and, finally, clinical outcomes by mRS at day 90. This paper includes consecutive patients with anterior circulation LVO treated from 1 January 2016 to 30 June 2018. For the LRG patients not repatriated immediately and RRG patients not repatriated within 24 hours, the reasons for same and outcomes are recorded. Details and outcomes of patients in either group who were readmitted back to the ESC are recorded.

The 90-day $\mathrm{mRS}$ was performed in the referring institution by the local stroke team, usually a clinical nurse specialist in stroke. The TICI assessments were verified by at least one other neuroradiologist, not involved in the procedure. If there was disagreement between readers, a third reader would evaluate the TICI score. This is done as standard procedure for all thrombectomy cases in our institution. Neither the mRS nor TICI assessments were blinded at the time as they are part of standard clinical care. 
Ischemic Stroke

\begin{tabular}{|c|c|c|c|c|}
\hline & All & Local & Remote & Sts \\
\hline Total patients & 435 & 352 & 83 & \\
\hline Male: female & $228: 207$ & 195:157 & $33: 50$ & \\
\hline Age & $70(20-101)$ & $70(20-101)$ & $71(32-94)$ & $P=0.857$ \\
\hline \multicolumn{5}{|l|}{ Baseline mRS } \\
\hline 0 & $413(95 \%)$ & $336(95 \%)$ & 77 (93\%) & \\
\hline 1 & $15(3 \%)$ & $11(3 \%)$ & $4(5 \%)$ & \\
\hline 2 & $1(0.3 \%)$ & 0 & $1(1 \%)$ & \\
\hline 3 & $2(0.7 \%)$ & $2(0.6 \%)$ & 0 & \\
\hline 4 & $4(1 \%)$ & $3(0.9 \%)$ & $1(1 \%)$ & \\
\hline $0-2$ & $429(99 \%)$ & 347 (99\%) & $83(99 \%)$ & \\
\hline $\begin{array}{l}\text { NIHSS } \\
\quad * 2 \text { intubated }\end{array}$ & $16(5-30)$ & $16(5-30)$ & $14(5-26)$ & $P=0.001$ \\
\hline ASPECTS & $9(5-10)$ & $9(5-10)$ & $9(5-10)$ & $P=0.857$ \\
\hline \multicolumn{5}{|l|}{ Collaterals } \\
\hline$<50 \%$ & $54(13 \%)$ & $50(15 \%)$ & $4(5 \%)$ & \\
\hline $50 \%-75 \%$ & $120(29 \%)$ & $104(30 \%)$ & $16(20 \%)$ & \\
\hline$>75 \%$ & $247(59 \%)$ & $188(55 \%)$ & $59(75 \%)$ & $P=0.001$ \\
\hline \multicolumn{5}{|l|}{ Occlusion site } \\
\hline MCA M1 & $260(60 \%)$ & 207 (59\%) & $53(64 \%)$ & \\
\hline MCA M2 & $47(11 \%)$ & $39(11 \%)$ & $8(10 \%)$ & \\
\hline ICA & $91(21 \%)$ & $77(22 \%)$ & $14(17 \%)$ & \\
\hline Tandem ICA+M1 & $30(7 \%)$ & $25(7 \%)$ & $5(6 \%)$ & \\
\hline Tandem ICA+M2 & $6(1 \%)$ & $4(1 \%)$ & $2(2 \%)$ & \\
\hline CCA & $1(0.2 \%)$ & 0 & $1(1 \%)$ & \\
\hline \multicolumn{2}{|c|}{$\begin{array}{l}\text { Thrombectomy rate/stroke diagnosis (as } \\
\text { per national stroke diagnoses numbers) }\end{array}$} & $6.50 \%$ & $2.50 \%$ & \\
\hline
\end{tabular}

Baseline data and outcome metrics for each group were evaluated. The groups are quite distinct by virtue of distance from the ESC and the primary purpose is not to compare them but to evaluate each group for safety of process. Some comparisons are made for illustrative purposes related to components of the pathway which should not necessarily differ. Comparisons were performed with Chi-square, Mann-Whitney and Kruskal-Wallis tests. The criterion for statistical significance was defined as $\mathrm{P}<0.05$.

\section{RESULTS}

A total of 435 patients underwent thrombectomy during this time period, with 352 transferred from the LRG and 83 transferred from the RRG. The baseline demographics for each group are described in table 1 . The overall median presenting NIHSS was 16 (5-30) with a median ASPECTS of 9 (5-10). Fifty-six per cent $(n=245)$ of patients received thrombolysis, 59\% (247) had collateral scores $\geq 75 \%$. Successful recanalisation with a TICI $2 b-3$ result was achieved in $88 \%$ of patients $(n=381)$. Two per cent $(n=8)$ were performed under general anaesthetic and the remainder were performed under local anaesthetic, with occasional use of conscious sedation as required. 5.5\% $(n=25)$ of patients included also received acute stenting at the time of thrombectomy. Good functional outcome defined as mRS of $0-2$ was seen in $47 \%$ of patients overall $(n=201)$.), table 2 . Key time metrics per group are evaluated in table 3. Locations of LRG and RRG PSC are outlined in figure 1. The LRG had a shorter door to CT time compared with the RRG, with a median of 28 mins

\begin{tabular}{lcccc}
\hline \multicolumn{4}{l}{ Table 2} & \multicolumn{2}{l}{ 90-day outcomes } & & \\
\hline mRS & \multicolumn{1}{l}{ All * } & Local region & Remote region & Sts \\
\hline 0 & $101(24 \%)$ & $75(22 \%)$ & $26(32 \%)$ & \\
1 & $65(15 \%)$ & $51(15 \%)$ & $14(17 \%)$ & \\
2 & $35(8 \%)$ & $28(8 \%)$ & $7(8 \%)$ & \\
3 & $48(11 \%)$ & $39(11 \%)$ & $9(11 \%)$ & \\
4 & $69(16 \%)$ & $54(16 \%)$ & $15(18 \%)$ & \\
5 & $28(7 \%)$ & $25(7 \%)$ & $3(4 \%)$ & \\
6 & $80(19 \%)$ & $72(21 \%)$ & $8(10 \%)$ & $\mathrm{P}=0.024$ \\
$0-2$ & $201(47 \%)$ & $154(45 \%)$ & $47(57 \%)$ & $\mathrm{P}=0.034$ \\
\hline
\end{tabular}

*nine patients lost to follow-up.

vs 39 mins, $(\mathrm{P}=0.003)$. Onset to thrombolysis was also shorter in this group (median 135 mins vs 165 mins, $\mathrm{P}=0.015$ ).

In the LRG, 91\% $(n=322)$ were repatriated to their referring institution immediately after the procedure. A total of 30 patients from the LRG (9\%) were admitted to the ESC. The median length of stay (LOS) was 7 days (range 2-87). The median NIHSS was 20 in this group compared with 16 in the group that was immediately repatriated $(\mathrm{P}=0.007)$, table 4 . They also had a lower median ASPECTS (8 vs 9; $\mathrm{P}=0.001$ ). The most common reasons for admission were the development of intracranial haemorrhage following thrombectomy $(n=10)$, the need for immediate ICU admission $(n=7)$ or decompressive craniectomy $(n=4)$. Good clinical outcome was seen in $20 \%$ of admitted patients compared with $47 \%$ in those immediately repatriated $(\mathrm{P}=0.006)$, with a mortality rate of $27 \%$ and $20 \%$ respectively $(\mathrm{P}=0.377)$.

In the RRG, $67 \%(n=56)$ were repatriated within 24 hours. A total of 27 patients (33\%) from the RRG were admitted to the ESC beyond the usual timeframe of 24 hours. The median LOS was 5 days for this group (range 3-15 days). Their presenting median NIHSS was slightly higher compared with those who were repatriated within 24 hours (16 vs $13 ; \mathrm{P}=0.075$ ), while the median ASPECTS was 8 and 9 respectively $(\mathrm{P}=0.711)$. The most common reason for admission included general observation $(n=7)$, intracranial haemorrhage post-EVT $(n=6)$ and the need for mechanical intubation either pre- or post-EVT $(n=5)$. Good clinical outcome was observed in $41 \%$ of those admitted compared with $65 \%$ in those immediately repatriated $(\mathrm{P}=0.042)$. Mortality rates were $22 \%$ and $4 \%$ respectively $(\mathrm{P}=0.007)$.

A total of 10 patients $(2 \%)$ required re-admission to the ESC after repatriation due to issues directly related to their stroke or thrombectomy. These included decompressive hemicraniectomy $(n=3)$, craniotomy and evacuation of intra-parenchymal haematoma $(n=1)$, cranioplasty $(n=1)$, removal of infected bone flap

\begin{tabular}{|c|c|c|c|}
\hline $\begin{array}{l}\text { Median times } \\
\text { (range) mins }\end{array}$ & $\begin{array}{l}\text { Local region } \\
\text { Ant circ } n=352\end{array}$ & $\begin{array}{l}\text { Remote region } \\
\text { Ant circ } n=83\end{array}$ & $\begin{array}{l}\text { Statistical analysis } \\
\text { Kruskal-Wallis }\end{array}$ \\
\hline Door to $\mathrm{CT}$ & $28(3-600)$ & $38(4-146)$ & $\mathrm{P}=0.003$ \\
\hline Onset to $\mathrm{CT}$ & $118(16-1229)$ & $136(10-998)$ & $\mathrm{P}=0.525$ \\
\hline Onset to $t-P A^{*} \dagger$ & $133(21-400)$ & $165(45-250)$ & $\mathrm{P}=0.015$ \\
\hline $\begin{array}{l}\text { Onset to groin } \\
\text { puncture }\end{array}$ & $256(46-1342)$ & $405(232-1320)$ & $\mathrm{P}=0.001$ \\
\hline $\begin{array}{l}\text { Onset to } \\
\text { reperfusion }\end{array}$ & $295(57-1360)$ & $431(241-1353)$ & $P=0.001$ \\
\hline
\end{tabular}




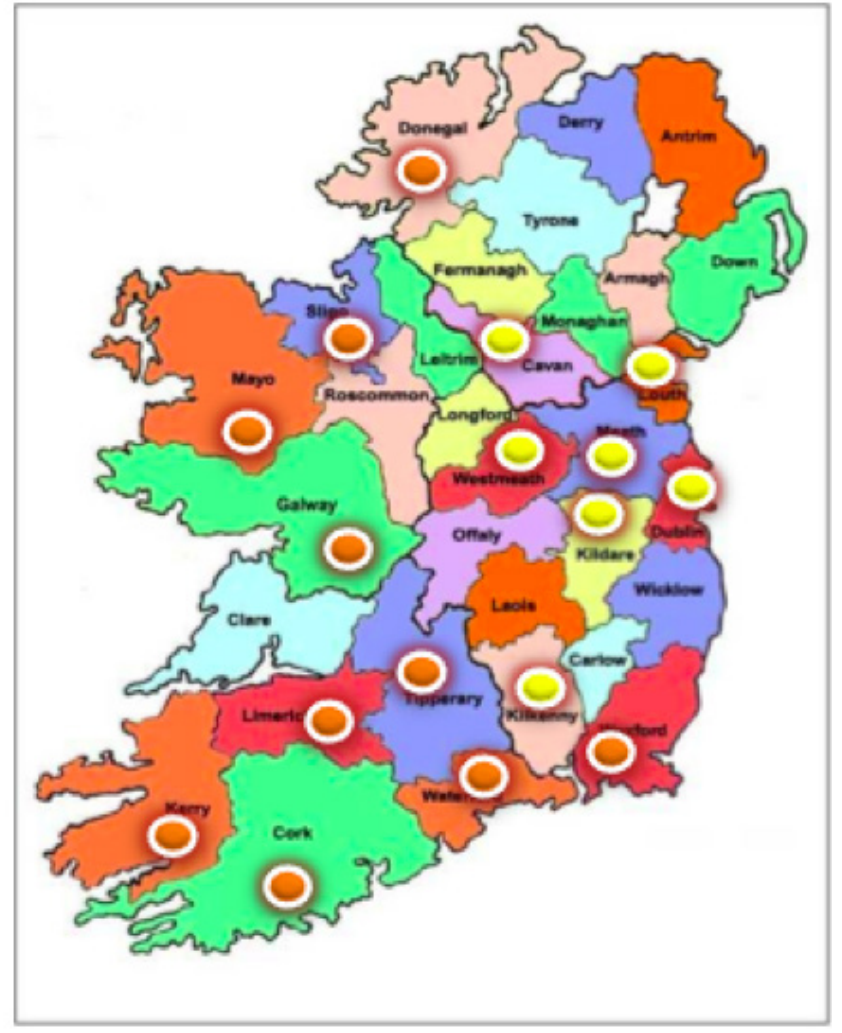

LRG

\section{RRG}

Figure 1 Location of PSC

$(n=1)$, carotid endarterectomy work-up $(n=1)$, carotid stenting $(n=1)$, femoral pseudoaneurysm post-EVT $(n=1)$ and subarachnoid haemorrhage $(n=1)$. Fifty per cent of these had functional independence (mRS 0-2) at 90 days with only one fatality.

In addition to those who underwent thrombectomy, 66 patients were transferred to the ESC but did not proceed to thrombectomy: 42 (64\%) from the LRG and 24 (36\%) from the RRG. This accounted for 11\% (42 of 394) from LRG and $22 \%$ (24 of 107) from RRG. Sixty-five per cent $(n=43)$ did not proceed due to recanalisation of previously demonstrated LVO, either at angiography or on repeat CTA or clinically improved on arrival. Thirty per cent $(n=20)$ (11 LRG, 9 RRG) were unsuitable because they had developed infarction. Three patients $(5 \%)$ overall were unsuitable due to the development of haemorrhage on repeat imaging.

\section{DISCUSSION}

The two most common systems of EVT provision are "drip and ship" and "mothership" models. A recent meta-analysis by Ciccone et al was unable to demonstrate conclusively which model was superior in terms of patient survival, favourable functional outcome and recanalisation. ${ }^{11}$ Computational modelling studies have found that a mothership model is effective when the distance between the PSC and the ESC is short or with more efficacious time to thrombolysis. ${ }^{12}$ However, with greater distances between the two centres, a drip and ship model becomes the optimum model of care. ${ }^{67}$ A study examining drip and ship vs direct to endovascular thrombectomy in Ireland found that a drip and ship model would be favoured if treatment and turnaround times are optimised. ${ }^{12}$ Due to logistical, organisational and infrastructure constraints of our system, we do not perform the mothership model for LVO AIS except for those patients in the ESC catchment area. Instead we consider the drip, ship, retrieve and leave model described herein as the most pragmatic and most appropriate model of care suited to the characteristics of the Irish healthcare system.

This system was developed as the ESC would not be capable of delivering the mothership model of care or keeping patients in the ESC, given existing resources. Similar to healthcare systems worldwide, in Ireland there have been significant funding pressures over the past decade in addition to overcrowding in emergency departments and lack of acute hospital beds. Ireland has the lowest number of acute hospital beds in the Organisation for Economic Co-operation and Development at 2.4 per 1000 of the population compared with the average of 3.1 per 1000 of the population in $2015 .^{13}$ These realities meant that a pragmatic system of care was necessary if Irish patients were to experience the huge improvements in outcomes following LVO AIS. Without this model of care, there would be no means of delivering EVT to LVO AIS patients. This system of care requires many partners across a wide range of specialties and services to work cohesively to deliver better quality stroke care for these patients with severe acute stroke. Clinical outcomes in our drip, ship, retrieve and leave system of care have clinical outcomes similar to those seen in other national stroke registries. In particular, we have shown good outcomes with low mortality rates in those that

\begin{tabular}{|c|c|c|c|c|c|c|}
\hline & $\begin{array}{l}\text { Admitted local region } \\
\text { group } n=30\end{array}$ & $\begin{array}{l}\text { Immediately repatriated local } \\
\text { region group } n=322\end{array}$ & Sts & $\begin{array}{l}\text { Admitted remote region } \\
\text { group } n=27\end{array}$ & $\begin{array}{l}\text { Immediately repatriated remote } \\
\text { region group } n=56\end{array}$ & Sts \\
\hline Male: female & $13: 17$ & $182: 140$ & & 10:17 & $23: 33$ & \\
\hline Age & 53 & 70.5 & & 70 & 72.5 & \\
\hline NIHSS & 20 & 16 & $P=0.007$ & 16 & 13 & $P=0.075$ \\
\hline$t-P A$ & $13(43 \%)$ & $182(57 \%)$ & $P=0.004$ & $11(41 \%)$ & 39 (70\%) & $\mathrm{P}=0.011$ \\
\hline \multicolumn{7}{|l|}{ Collaterals } \\
\hline$<50 \%$ & $9(30 \%)$ & $41(13 \%)$ & & $1(4 \%)$ & $3(5 \%)$ & \\
\hline $50-75 \%$ & $5(17 \%)$ & 99 (31\%) & & $5(19 \%)$ & $11(20 \%)$ & \\
\hline$>75 \%$ & $13(43 \%)$ & $175(56 \%)$ & $P=0.247$ & $18(67 \%)$ & 41 (75\%) & $P=0.537$ \\
\hline \multicolumn{7}{|l|}{$\mathrm{mRS}$} \\
\hline
\end{tabular}


are immediately repatriated to their referring institution. Our overall rate of good clinical outcomes is $47 \%$, which is comparable to the $46 \%$ rate derived from the HERMES meta-analysis, while our mortality rate was $19 \%$ compared with $15.3 \%{ }^{1}$

These rates compare favourably compared with other national registries. The Mr CLEAN Registry in the Netherlands evaluating EVT in routine clinical practice reported mortality rates of $29 \%$ with good clinical outcome in $37.9 \% .{ }^{14}$ Goyal et al performed a multi-centre study on the safety and efficacy of thrombectomy for patients not meeting top-tier evidence trial criteria $50 \%$ of patients would have been denied if top-tier guidelines were strictly followed). They reported good clinical outcome in $39 \%$, with a mortality rate of $30 \% .{ }^{15}$ Wollenweber et al evaluated more than 2000 patients in the German Stroke Registry from 25 sites. For anterior circulation patients only, they reported a mortality rate of $29 \%$, with good clinical outcome in $37 \%$ of cases. ${ }^{16}$ The NASA and TRACK registries evaluated real-world stent-retriever thrombectomy for AIS for those presenting within and beyond 6 hours of onset and considered good outcomes and mortality rates separately depending on symptom onset time. Those presenting 16-24 hours' post-onset of symptoms had a mortality rate of $33.3 \%$ as compared with $21.6 \%$ presenting $6-16$ hours and $20.6 \%$ if presenting $0-6$ hours post-onset. ${ }^{17}$ Good outcomes were seen in $38.9 \%, 46.2 \%$ and $48 \%$ for these varied timeframes ${ }^{17}$. These rates are comparable to our overall mortality rate of $19 \%$ and good outcome of $47 \%$ including patients presenting up to 24 hours' post-onset of symptoms.

Counter intuitively, patients referred from more remote PSC's have a trend towards better outcomes than patients referred from LRG hospitals, even though the remote group also had significantly longer onset to treatment times for thrombolysis and EVT (table 3). The RRG had significantly less severe stroke symptoms on admission compared with the LRG (NIHSS 16 vs 14, collaterals $>75 \% \mathrm{P}=0.001$; table 1 ). The current study did not set out to compare outcomes between these two groups of patients. Patients from the RRG are more highly selected although not by design. We know that a smaller percentage of stroke patients are referred from this group compared to the LRG $(2.5 \%$ vs $6.5 \%$ (thrombectomy rate/stroke diagnosis)). There may be an unintentional selection bias with only the best candidates being referred. In addition, due to the distance from the ESC, they are more likely to be unsuitable for thrombectomy by the time they arrive at the ESC with only those with very good collaterals remaining suitable. Overall, $8 \%$ of patients transferred for thrombectomy from the RRG were unsuitable due to established infarct compared to 3\% in the LRG. Transfer times in the remote region group in our study do not have an adverse effect on patient outcomes, compared with published trial outcomes. However, the incidence of patients becoming unsuitable during the transfer period is not taken into consideration here.

Ninety-one per cent of patients referred from local region hospitals did not require any admission to the ESC. The remaining $9 \%$ of them have a median length stay of 7 days. For the remote region group, 33\% of patients stay longer than the intended 24 hours, resulting in a median length of stay of 5 days. These figures may help estimate bed requirements in planning for a regional ESC service if this model of care is chosen. Previous studies have reported a 30-day re-admission rate post-thrombectomy of $12.5 \%$ with the most common reasons for re-admission including infections, cardiac causes or recurrent stroke or transient ischaemic attack. This is much higher than our study, where only $2 \%$ of patients were re-admitted to the ESC. With regards to these patients, we are not aware of any delay or increased morbidity due to PSC inexperience in managing thrombectomy patients. However, this only refers to patients re-admitted to our ESC. We have not evaluated for patients who may have required re-admission to their own PSC post-discharge.

The repatriation of patients back to the referring RRG is done within the constraints of our health service and is not always under our control. LRG hospitals are required to take patients back immediately in order for the service to work and so they only stay if we feel it is necessary for them to stay. Once a patient is admitted to our hospital, that is, all of the RRG, they are no longer a high priority transfer case and there can be a delay in arranging the ambulance services to return the patient. Furthermore, if there are no available beds in the referring institution, a patient cannot be immediately repatriated. In this situation, patients can often remain in the ESC for further observation and to commence/continue investigations as part of the routine stroke work-up. We believe the improved outcome for those repatriated, as per intention, indicates that we are correctly identifying those patients who are suitable for transfer and that they are not suffering because of this care pathway.

The centralisation model of all stroke treatment has positive and negative effects. The logistics of actually implementing this service is challenging, with negative knock-on effects and significant resource implications in both the ESC and PSC. A UK study published in 2018 found that redirecting patients to the ESC reduces time to thrombectomy with only a 15 -minute delay in thrombolysis ${ }^{18}$. This model is applicable for LVO AIS where thrombolysis has a minimal effect. However, it is not the most appropriate model for non-LVO AIS which make up the majority of acute strokes. They suggest that a mixed model of ESC with PSC is necessary due to the large increase in the number of stroke patients being admitted to the $\mathrm{ESC}^{18}$. Centralising acute stroke services has beneficial effects on numbers of patients receiving interventions, but this varies according to model employed ${ }^{19}$. Our early repatriation model is not dependent on the model of patient entry but does reduce the impact on the ESC, by not having to keep EVT patients. It requires the ability of the referring PSCs to maintain their ability to manage acute stroke patients whether or not they are transferred for thrombectomy. Currently in Ireland, we have 24 PSCs giving us a rate of 4.2 stroke units per million inhabitants. We believe that the early repatriation approach enables the treatment of large numbers of patients optimising the use of existing stroke services in referring PSCs.

The uptake of thrombectomy is growing worldwide but there is a long way to go. The European rate of thrombectomy for 2016 is merely $1.9 \%$ with lack of expertise, lack of facilities and costs being the main reasons for not providing a thrombectomy service $^{20}$. If we compare our service to the UK, which could be regarded as a similar health system, we are performing more than six times the number of EVT procedures per million inhabitants.

To our knowledge, this is the first description of the drip, ship, retrieve and leave model. The early post-procedure period requires close observation. These include stroke-specific considerations including blood pressure and glucose control, and procedure-related complications including access-site haematomas and pseudoaneurysm. Comparing outcomes of those who return within the proposed timeline and to those who require additional admission to our stroke unit, we have been able to correctly predict which patients should remain in the ESC for access to a further stroke physician, neuroradiology or neurosurgery intervention. For the remainder, who have an uncomplicated early post-procedural period, our patients are quickly 
back in their PSC where they are managed by the same stroke team who would have managed them if they have not had EVT. Our mortality rates are comparable or lower for those who are immediately repatriated, when compared with international stroke registries, indicating that this is a safe method of delivering endovascular thrombectomy within the confines of our stroke network.

\section{CONCLUSION}

The immediate or early repatriation of patients following transfer for thrombectomy from local and remote regional PSCs with the drip, ship, retrieve and leave model provides clinical outcomes comparable to, or better than, previously reported trials of EVT. This enables the treatment of larger numbers of patients in a system of care which would not otherwise facilitate this treatment. The impact on the resources required within the ESC is mitigated and the continued use of pre-existing stroke services in non-endovascular-capable PSC is optimised. Within a changing evidence base and expanding demand for thrombectomy services, this model offers an effective option for resourcelimited services.

\author{
Author affiliations \\ ${ }^{1}$ Interventional Neuroradiology Service, Department of Radiology, Beaumont \\ Hospital, Dublin 9, Ireland \\ ${ }^{2}$ Royal College of Surgeons, Dublin 2, Ireland \\ ${ }^{3}$ Department of Geriatric and Stroke Medicine, Mater Misericordiae University \\ Hospital, Dublin 7, Ireland \\ ${ }^{4} U C D$ School of Medicine, Dublin, Ireland \\ ${ }^{5}$ Department of Geriatric and Stroke Medicine, Beaumont Hospital, Dublin 9, Ireland \\ ${ }^{6}$ Department of Geriatric and Stroke Medicine, Tallaght University Hospital, Dublin \\ 24, Ireland \\ ${ }^{7}$ Department of Geriatric and Stroke Medicine, Connolly Hospital Blanchardstown, \\ Dublin 15, Ireland \\ ${ }^{8}$ Department of Geriatric and Stroke Medicine, St Vincent's University Hospital, Dublin \\ 4, Ireland \\ 'Department of Geriatric and Stroke Medicine, St. James' Hospital, Dublin 3, Ireland \\ ${ }^{10}$ Department of Geriatric and Stroke Medicine, University Hospital Limerick, \\ Limerick, Ireland
}

Acknowledgements The authors wish to express their gratitude to Caroline Deegan, Imelda Noone, Mary-Kate Meagher, Paul O’Brien, Patricia Daly, Roisin Kelly, Suzanne Greene, Nicola Cogan, Fiona Connaughton, Olwyn Lynch, Clare Fallon, Sinead Gallagher, Mary Flanagan, Paul Cotter, Hannah Murugan, Gerard Murugasu, Frances Smith, Lisa Donaghy, Nora Cunningham, Tom O'Malley, Niamh Murtagh, Paula Hickey, Una Moffatt, Ken Mulpeter, Christine McLaughlin, Thomas Walsh, Tish Galvin, Riona Mulcahy, Breda Jones, Eithne Harkin, Elaine Crosby, Simon Cronin and Glen Arrigan for their contribution to this paper.

Contributors All authors contributed to the manuscript in the following areas: 1. Substantial contributions to the conception and design of the work and the acquisition of the data. 2. Critically drafting and revising the work. 3. Final approval of the version to be published. 4 . Agreement to be accountable for all aspects of the work.

Funding The authors have not declared a specific grant for this research from any funding agency in the public, commercial or not-for-profit sectors.
Competing interests None declared.

Patient consent for publication Not required.

Provenance and peer review Not commissioned; externally peer reviewed.

Data availability statement Data are available upon reasonable request.

\section{REFERENCES}

1 Goyal M, Menon BK, van Zwam WH, et al. Endovascular thrombectomy after large-vessel ischaemic stroke: a meta-analysis of individual patient data from five randomised trials. Lancet 2016;387:1723-31.

2 Nogueira RG, Jadhav AP, Haussen DC, et al. Thrombectomy 6 to 24 hours after stroke with a mismatch between deficit and infarct. N Engl J Med 2018;378-11-21.

3 Albers GW, Marks MP, Kemp S, et al. Thrombectomy for stroke at 6 to 16 hours with selection by perfusion imaging. N Eng/ J Med 2018;378:708-18.

4 Saver JL, Goyal M, van der Lugt A, et al. Time to treatment with endovascular thrombectomy and outcomes from ischemic stroke: a meta-analysis. JAMA 2016;316:1279-88.

5 Menon BK, Sajobi TT, Zhang Y, et al. Analysis of workflow and time to treatment on thrombectomy outcome in the endovascular treatment for small core and proximal occlusion ischemic stroke (escape) randomized, controlled trial. Circulation 2016;133:2279-86.

6 Holodinsky JK, Williamson TS, Kamal N, et al. Drip and ship versus direct to comprehensive stroke center: conditional probability modeling. Stroke 2017:48:233-8.

7 Milne MSW, Holodinsky JK, Hill MD, et al. Drip 'n ship versus mothership for endovascular treatment: modeling the best transportation options for optimal outcomes. Stroke 2017;48:791-4.

8 Motyer R, Thornton J, Power S, et al. Endovascular thrombectomy beyond 12 hours of stroke onset: a stroke network's experience of late intervention. J Neurointerv Surg 2018;10:1043-6.

9 Motyer R, Asadi $\mathrm{H}$, Thornton J, et al. Current evidence for endovascular therapy in stroke and remaining uncertainties. J Intern Med 2018;283:2-15.

10 O'Neill D, Griffin E, Doyle KM, et al. A standardized aspiration-first approach for thrombectomy to increase speed and improve recanalization rates. AJNR Am J Neuroradiol 2019:40:1335-41.

11 Ciccone A, Berge E, Fischer U. Systematic review of organizational models for intraarterial treatment of acute ischemic stroke. Int J Stroke 2019;14:12-22.

12 Holodinsky JK, Patel AB, Thornton J, et al. Drip and ship versus direct to endovascular thrombectomy: the impact of treatment times on transport decision-making. Eur Stroke J 2018:3:126-35.

13 Independent.ie. Available: https://www.independent.ie/irish-news/health/trolley-crisishealth-minister-says-number-of-patients-on-trolleys-is-not-acceptable-36451440.htm

14 Jansen IGH, Mulder MJHL, Goldhoorn R-JB, et al. Endovascular treatment for acute ischaemic stroke in routine clinical practice: prospective, observational cohort study (Mr Clean Registry). BMJ 2018;360:k949.

15 Goyal N, Tsivgoulis G, Frei D, et al. A multicenter study of the safety and effectiveness of mechanical thrombectomy for patients with acute ischemic stroke not meeting top-tier evidence criteria. J Neurointerv Surg 2018;10:10-16.

16 Wollenweber FA, Tiedt S, Alegiani A, et al. Functional outcome following stroke thrombectomy in clinical practice. Stroke 2019;50:2500-6.

17 Mokin M, Abou-Chebl A, Castonguay AC, et al. Real-world stent retriever thrombectomy for acute ischemic stroke beyond 6 hours of onset: analysis of the NASA and track registries. J Neurointerv Surg 2019;11:334-7.

18 Allen M, Pearn K, James M, et al. Maximising access to thrombectomy services for stroke in England: a modelling study. Eur Stroke J 2019;4:39-49.

19 Ramsay AIG, Morris S, Hoffman A, et al. Effects of centralizing acute stroke services on stroke care provision in two large metropolitan areas in England. Stroke 2015;46:2244-51.

20 Aguiar de Sousa D, von Martial R, Abilleira S, et al. Access to and delivery of acute ischaemic stroke treatments: a survey of national scientific societies and stroke experts in 44 European countries. Eur Stroke J 2019;4:13-28. 